\title{
Limbo
}

\section{Der erste Kreis der Hölle}

Erschienen in: WORTATLAS

Von: Simon Godart

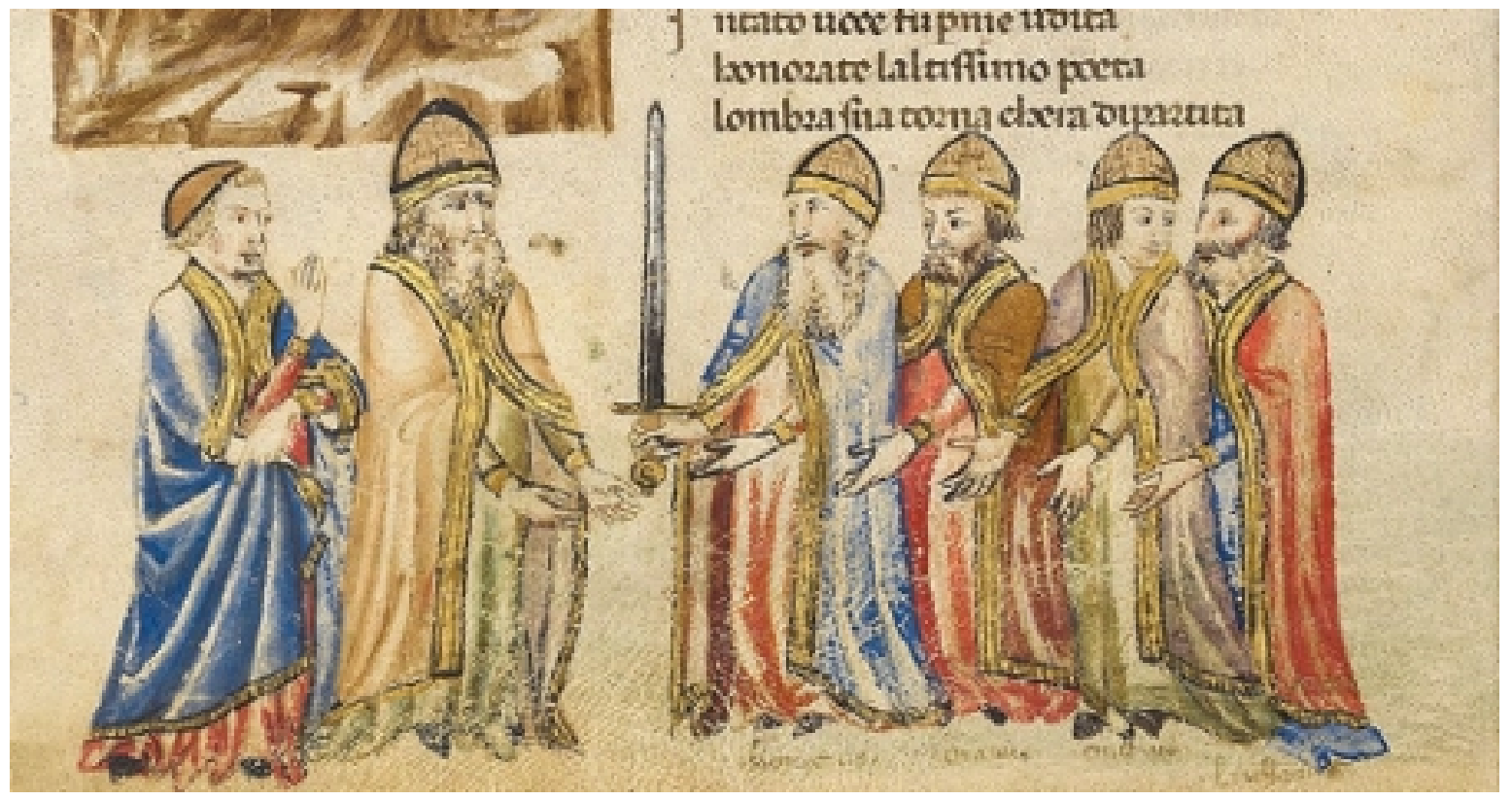

Abbildung 1: Virgil und Dante im Limbus, Codex Altonensis, Wikimedia Common

Das Jahr 2021 ist zufälligerweise beides geworden: Einerseits ist es das Dante-Jahr (am 14. September 1321 jährt sich sein siebenhundertster Todestag), und andererseits das nunmehr zweite Covid-Jahr. Dieser Zufall ist sicher nur eine jener historischen Konstellationen, denen man keine große Bedeutung zumessen sollte. Beim ersten Lockdown und der globalen Schockstarre zu Beginn des Frühjahrs im vergangenen Jahr hätte es zwar nicht wegen eines Jubiläums gepasst, thematisch dafür aber umso mehr, an Boccaccio zu denken: Der Rückzug aus dem öffentlichen Leben hat doch zu deutlich an die Rahmenhandlung des Decamerone erinnert. Ein beklemmend langes Jahr später wird es schwieriger, dem Zufall zu entsprechen und eine Beziehung zu erkennen zwischen dem Dichter der Göttlichen Komödie und dem Alltag im Homeoffice. Dantes epochemachende Jenseitsfahrt hat mit dem Alltag im Homeoffice nicht viel gemein auch wenn seit Dan Browns Beststeller „Inferno“ von 2013 (und dem gleichnamigen Spielfilm von 2016) der Dichter in den Ruf geraten sein könnte, etwas mit bedrohlichen Seuchen zu tun zu haben. „Lasst, die ihr eintretet, alle Hoffnung fahren“ (Dante, Inf. III, v. 9), wie am Eingang der Hölle zu lesen ist, taugt trotz des ebenfalls viral um sich 
greifenden Zynismus wenig als Motto für 2021 - und es wäre zudem wenig ratsam, sich in die laufende Debatte um Dantes Wert als Weltliteratur auch nur aus der Ferne einzuschalten.

Wenn man aber vom heimischen Schreibtisch gedanklich an die „Orte der Universität" wandert, gibt es einen unter ihnen, der im Zuge jüngerer Umgestaltungen eine leise Beziehung zu den Eingangsgesängen der Göttlichen Komödie unterhalten könnte - die Universitätsbibliothek. Nicht aus dem zu offensichtlichen Grund, dass dies allgemein der Ort ist, an dem man Klassiker der Weltliteratur vermuten würde. Man muss dabei nicht an schwere Lederbände und indirekt beleuchtete Lesesäle denken; ähnlich wie in Markus Steinmayrs Nachdenken über die „Vorzimmer“ der Universität ist es das Foyer selbst, das an Dante erinnert.

Wer nun glaubt, dass Dantes Ruhm als epochaler Dichter durch Verbannung ins Vorzimmer weiter in Gefahr geraten soll, irrt; er soll zweifelsfrei, in hoher Exemplarzahl und möglichst komfortabel im Lesesaal verbleiben. Aber es wird seinem Ruhm nicht schaden, wenn er, auch nur durch eine lose Assoziation, auch außerhalb von diesem ein wenig weiterwirken darf. Was hat er also im Foyer zu suchen? Es ist eine Trivialität, von technischer Neuerung elektronischer Schließfächer bewirkt, wie sie in den vergangenen Jahren in vielen Bibliotheken eingeführt wurden. Man hat die Münzschließfächer mit ihren kleinen Schlüsseln gegen anspruchsvollere und nutzer*innenfreundlichere Mechanismen ausgetauscht; die Digitalisierung macht auch vor den Schließfächern nicht halt. Hat man früher eine Münze einwerfen müssen, um dann den Schlüssel abzuziehen und mit sich zu führen, wählt man nun einen Pin oder verwendet einen entsprechenden elektrischen Transponder (im Schlüssel oder im Studierendenausweis), und der Spind verschließt sich wie von Zauberhand. Es ist sicher erfreulich, nicht länger auf Münzen angewiesen zu sein, um sich eingangs seiner Habseligkeiten zu entledigen - man kann nun dank der Technik auf den Obolus verzichten, der oft nicht zu leicht aufzutreiben gewesen ist. ${ }^{1}$ Doch hat dieser kleine Fortschritt auch seine Tücken. Wenn man digital und kontaktlos sein Spindabteil verriegeln kann, fehlt nun dasjenige, was man früher für den Zeitraum des Aufenthalts in der Tasche mit sich tragen musste - der Spindschlüssel, der mit dem ungemeinen Vorzug aufwarten konnte, dass die Nummer des Schließfachs auf ihm verzeichnet gewesen ist. Beim Verlassen führte einen diese Nummer also mit Leichtigkeit zum Ausgangspunkt zurück, derart trivial, dass man sich dessen vielleicht nie recht bewusst geworden ist. Entfernt man die Schlüssel, entfernt man auch dieses Zeichen - und beschwört kleine, aber lästige Identitätskrisen bei den Nutzer*innen herauf, die nicht mehr wissen, wohin mit sich, also: wo sie ihre sieben Sachen haben. Um dieser Verirrung zu entgehen, war es notwendig geworden, sich die Kennziffer des Schrankes ohne jedes Hilfsmittel zu merken - was durchaus eine Herausforderung darzustellen schien, insbesondere für diejenigen, die für Stunden über Büchern gesessen hatten und bei all dem Neuen und Einsichtsvollen, das sie ihnen entnehmen konnten, die Nummer ihres Spindes schlicht vergessen hatten. Beladen mit den zugelassenen Gerätschaften für die kommende Lektüre notiert sich eine Kennziffer nicht ohne weiteres, und man soll 
sogar manche dabei beobachtet haben, die Nummer einhändig mit dem Mobiltelefon abfotografiert zu haben, in weiser Voraussicht, dass man die Zahlenkombination in wenigen Momenten - vergessen haben wird.

Diese Unauffindbarkeit des eigenen Schließfachs ist keine Nebensache für den täglichen Umgang mit Bibliotheken. Man kann die Bücher den Menschen nicht einfach so zumuten, aber ebenso wenig den Büchern die Menschen; es braucht eine durchaus permeable, aber vor allem übersichtliche Schranke zwischen Innen und Außen, damit eine Bibliothek funktionieren kann. Die Institution verdankt dieser Schwellenzone mit gewissem Recht ihren Namen; die Bibliothek kann sich begriffsgeschichtlich nicht nur als biblíon théke, als Bücherkiste oder-kammer, sondern auch als

Ausleihtheke ${ }^{2}$ verstehen, als Tresen des Archivs, den man mitunter passieren darf. Und wirklich zählt die sanfte Kontrolle von Ein- und Ausgängen - von Büchern wie auch von Menschen, versteht sich - zu den wichtigsten Aufgaben der Institution. Es mag ein vollkommen pragmatisches Ritual sein, das die Nutzung der Räume daran bindet, sie ohne den eigenen weltlichen Besitz und geradezu transparent zu betreten, und darin aufgehen, den Bestand vor Verschmutzungen und Entwendungen zu schützen. Aber es hat doch etwas von einem rite de passage, dem keine Störungen zuzumuten sind weshalb die Strapazierfähigkeit der Erinnerungskraft der Nutzer*innen mit den nackten Zahlencodes Gefahr läuft, den Betrieb ins Stocken zu bringen.

Die Bibliotheken sahen sich also vor ein Problem der Mnemotechnik gestellt. Die Lösung lag, wenn nicht auf der Hand, so in den Katalogen; die nüchternen, bürokratischen Kennziffern der Spinde wurden um die Namen von Figuren, Autor*innen und Forscher*innen ergänzt, die prominent und weithin lesbar auf die Spindtüren geschrieben wurden. Der zugrundeliegende Gedanke war, dass sich Personennamen dem Gedächtnis weit besser und leichter einprägen als anonyme Zifferfolgen - und so ist es möglich geworden, das eigene Hab und Gut etwa bei Hannah Arendt, Hamlet und vielleicht auch bei Dante Alighieri abzustellen. Die Staatsbibliothek Hamburg hat in unterschiedlichen Abteilungen beides unternommen und einmal Figuren-, einmal Forscher*innennamen in die Foyergestaltung aufgenommen - hier prangt beispielsweise einerseits blutrot Dracula im Vorraum, in Nachbarschaft von Hamlet und Momo, andernorts findet man bei Aby Warbug, Emmy Noether oder Peter Szondi wieder zu seinen Sachen. Die Auswahl der Benennungen erfolgte durch eine öffentliche Umfrage - Nutzer*innen wurden um Vorschläge gebeten, die dann auch in Überfülle eingingen und sich in Konkurrenz durchsetzen mussten.

Die Universitätsbibliothek der Freien Universität Berlin hat das Konzept übernommen, allerdings weniger auf Basisdemokratie als auf Korrespondenz geachtet - und den zweihundert Schrankfächern der Foyers die Namen aus dem hauseigenen Katalog zugeordnet. Die Mitarbeiter*innen des großen Lesesaals haben aus den Autor*innen ausgewählt, die dort prominent für die Lektüre verfügbar sind - wer innen mit einem Gesamtwerk vertreten ist, darf außen einen Spind bekleiden. Derart wurde die Entscheidung über die Namensgebung aufs Kerngeschäft des Archivs selbst ausgelagert, und die anonymen, kollektiven Prozesse der Edition und Publikation, des Ankaufs und der Kanonisierung haben sich so in der Foyergestaltung niedergeschlagen. 


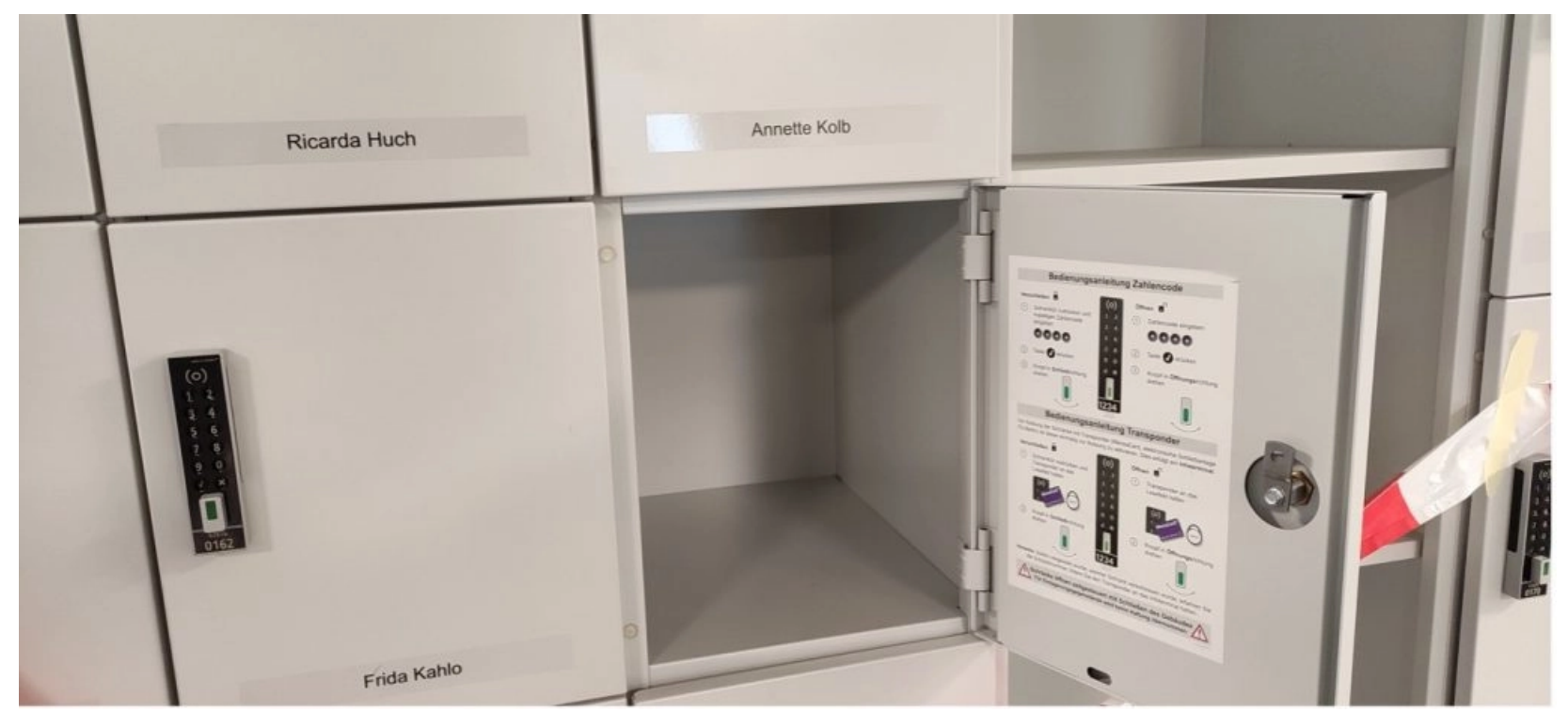

Abbildung 2: Universitätsbibliothek der Freien Universität Berlin, Foto Matheo Mareski @

Die Universitätsbibliothek hat so die stille Ehrfurcht vor dem archivierten Intertext ins Raumkonzept übersetzt. Nichts könnte für einen Ort der Bewahrung kultureller Schätze näherliegen. Dem Kanon kommt so eine neue, aber vertraute Funktion zu; seine Namen geben Orientierung. Es handelt sich schließlich um unvergessliche Namen - jede dieser Personen entstammt einem kanonischen, kulturellen und kollektiven Bewusstsein und bietet sich nur darum als lebenspraktische Orientierungsstütze an. Wer Hannah Arendtangeschrieben sieht, wird sich an die historische Person und ebenso an ihr Werk erinnern, wie es im Lesesaal unter der entsprechenden Kennziffer zu finden sein wird; und damit vielleicht auch sogleich an ihr Nachdenken über das Handeln, über Eichmann und über die totale Herrschaft usw. denken. Wenn man die Autorin denn schon kennt andernfalls beginnt die Lektüre vielleicht schon hier am Eingang eben der Institution, in der man ihre Texte finden kann. In diese äußerst ökonomische Signifikanz möglicher Implikationen kann die Kennzeichnung eines physischen Ortes leicht hineingenommen werden - fast so, als würde man die Mnemotechnik der klassischen Rhetorik ins Gegenteil wenden, sind es nun die bloßen Worte, die einen an die nicht nur gedachten, sondern wirklichen Orte zurückführen. Wenn bspw. im dritten Buch der Rhetorica ad Herennium die Technik erläutert wird, ${ }^{3}$ die Worte der Rede, die man zu halten hat, gedanklich an einzelnen, wohlbestimmten Plätzen eines inneren Hauses abzulegen und so beim Sprechen in Gedanken die Orte abzuschreiten, um über die Architektur zu den Worten zu finden, hat sich die Bibliotheksverwaltung entschieden, ihre Kataloge zu Markierungen zu machen, und so das Vorzimmer in einen tatsächlichen Gedächtnispalast, ${ }^{4}$ wie inn sich Matteo Ricci 1596 ausgedacht hatte, zu verwandeln, doch in gegenteiliger Funktion; die Namen leiten uns sicher aus der Welt der Worte und Zeichen zurück an ihre Schwelle und lassen uns so die richtige Stelle im Großbau der Bibliothek wiederfinden.

Die kanonischen Namen stammen dabei, so hat man es eingerichtet, nicht von lebenden Personen, und sie bilden einen gestalterischen ersten, äußersten Kreis der Bibliothek, durch den man - gleichermaßen notgedrungen, bürokratisch und ehrfürchtig - hindurch muss. Dass in dieser Kombination aus verfeinerter Mnemotechnik und Vorlagerung der großen Namen der Literatur- und Philosophiegeschichte das Archiv 
sich selbst eine kleine Allegorie erlaubt hat, mag dem Modell nach nur bedingt etwas mit Dante zu tun haben. Aber dennoch kann man schwer übersehen, dass die lange Reihe von großen, bedeutenden Persönlichkeiten gewissermaßen einen ,ersten Kreis der Hölle‘ darstellt - als Limbus auctorum sind hier wie bei Dante die großen Dichter*innen, Denker*innen und historischen Persönlichkeiten der Vorzeit versammelt.

Man schreibt im Homeoffice allein, und man vermisst die Bibliotheken nicht nur, weil man Informationen und Materialien in ihnen vermutet, die gerade fehlen - man vermisst sie auch als "dritte Orte“. So lautet der terminus technicus des Raumsoziologen Ray Oldenburg für Orte, die weder das Private des eigenen Wohnortes, noch das Öffentliche des Arbeitsplatzes haben, sondern „great good places“ sind: offene, soziale Orte, "offering both the basis of community and the celebration of it“. ${ }^{5}$ Dieses Konzept wurde in der gegenwärtigen Diskussion über das Selbstverständnis der Bibliotheken im Angesicht von Digitalisierung und freier Verfügbarkeit von Wissensinhalten prominent aufgenommen - sie sind Orte des Zusammenseins mit Anderen, des sozialen Lebens, oder sollen es zumindest werden. ${ }^{6}$ Ob Umgestaltungen wie die hier beschriebene in ihrer Abkehr von den nackten, anonymen Zahlen und ihrer Hinwendung zu den großen Namen der Vergangenheit an diesem Trend unmittelbar partizipieren, lässt sich schwer abschätzen. An heimischen Schreibtischen ist die Sehnsucht nach ,dritten Orten“ beständig größer geworden, auch wenn man dort wohl bis auf weiteres nicht nur am Schließfach Kontaktarmut zu schätzen wissen wird. Vielleicht tut es für den Moment auch eine virtuelle, kanonische Bekanntschaft auf Zeit, so wie Dante beim Abstieg in die Hölle nicht ohne seinen versierten Fremdenführer Vergil ausgekommen wäre. Die Gemeinschaft der eingeprägten Namen im transitorischen Bereich bildet ebenfalls einen Limbus, der nicht nur einen Kreis um die Magazine und Lesesäle umfasst, sondern den Eintretenden auch eine*n Gefährt*in aus ihrer Mitte an die Hand gibt, der*die sie für eine Zeit begleiten soll. In der Hoffnung, den eigenen Weg zu finden - und wieder zurück. Ob Vergils Name selbst einen der Spinde ziert, wird sich dann, wenn es wieder geht, vor Ort ergeben.

\section{References}

1. Um im Bild zu bleiben - im dritten Gesang der Göttlichen Komödie nutzen Vergil und Dante Charons Dienste zur Überquerung des Acheron gegen den Protest des mythischen Greises und zudem ohne den obligatorischen Charonspfennig zu leisten. Siehe Dante, Inf. 3, vv. 81-99.

2. Klaus Gantert, Bibliothekarisches Grundwissen, Berlin: De Gruyter Saur 2016 , S. 59. https://doi.org/10.1515/9783110321500.

3. Rhetorica ad Herennium : lateinisch-deutsch, hrsg. und übers. v. Theodor Nüßlein, München (u.a.): Artemis \& Winkler 1994, III 16, S. 164-171.

4. Vgl. Jonathan D. Spence, The Memory Palace of Matteo Ricci, New York (u.a.): Penguin Books 1985, S. 1-23.

5. Ray Oldenburg, The great good place : cafés, coffee shops, bookstores, bars, hair salons, and other hangouts at the heart of a community, Cambridge: Da Capo Press 1999, S. 14. 
6. So erklärt bspw. Klaus Ulrich Werner einleitend (Klaus Ulrich Werner, „Bibliothek als Ort", in: Praxishandbuch Bibliotheksbau: Planung - Gestaltung - Betrieb, hrsg. v. ders. u. Petra Hauke, Berlin/Boston: De Gruyter Saur 2016, S. 95-107, hier S. 7): „Denkraum' (Aby Warburg), gemeinsamer studentischer Lernort und öffentliches Wohnzimmer - die Renaissance der Bibliothek im digitalen Zeitalter ist eine Wiederentdeckung der Bibliothek als Raum und eine radikale Transformation vom Buchorientierten Wissensspeicher zum Nutzer-orientierten ,Dritten Ort': Immer weniger Besucher kommen in die Bibliothek lediglich um Medien auszuleihen.

SUGGESTED CITATION: Godart, Simon: Limbo. Der erste Kreis der Hölle, in: KWIBLOG, [https://blog.kulturwissenschaften.de/limbo/], 10.05.2021

DOI: https://doi.org/10.37189/kwi-blog/20210510-0830 


\section{DuEPublico}

Duisburg-Essen Publications online
UNIVERSITÄT
DEU SISER R G

offen im Denken

Dieser Text wird via DuEPublico, dem Dokumenten- und Publikationsserver der Universität Duisburg-Essen, zur Verfügung gestellt. Die hier veröffentlichte Version der E-Publikation kann von einer eventuell ebenfalls veröffentlichten Verlagsversion abweichen.

DOI: $\quad 10.37189 / \mathrm{kwi}-\mathrm{blog} / 20210510-0830$

URN: urn:nbn:de:hbz:464-20210510-103930-0

Alle Rechte vorbehalten. 